\title{
Organic matter mineralization in redox-dynamic environments: how does the redox reactivity of particulate electron acceptors affect microbial respiration rates?
}

\author{
MERET AEPPLI ${ }^{1}$, CHRISTIAN DEWEY ${ }^{2}$ AND SCOTT \\ FENDORF $^{1}$ \\ ${ }^{1}$ Stanford University \\ ${ }^{2}$ Lawrence Berkeley National Laboratory \\ Presenting Author: meret.aeppli@stanford.edu
}

Floodplains are active sites for transport, transformation, and storage of carbon and therefore play a critical role in the global carbon cycle. The structured porous media of floodplain soils coupled with dynamic hydrological states result in a heterogeneity in redox environments. This heterogeneity is reflected in variations of microbial metabolic pathways for organic matter mineralization. Further, organic matter mineralization is particularly responsive to ongoing and impending climate and land use changes. To predict how carbon turnover in floodplains will change in the future, we thus need to better understand the controls on organic matter mineralization.

Here, we focus on energetic constraints on organic matter mineralization under oxygen-depleted conditions. Particulate terminal electron acceptors (TEAs), including iron minerals, are often used in microbial respiration under these conditions and the redox reactivity of these TEAs potentially limits microbial respiration rates. We discuss relationships between the redox reactivity of particulate TEAs and soil organic matter mineralization for soils from the Slate and East River floodplains, Colorado, U.S.A. Soils from two sites and various depths differing in redox conditions were examined. We used a mediated electrochemical approach to quantify microbial reduction of particulate TEAs during anoxic soil incubations and to characterize the redox reactivity of these TEAs. Soils that were oxygen-depleted in the field showed no microbial reduction of particulate TEAs and low reactivity toward reduction of the TEAs in anoxic incubations; no carbon dioxide was produced. In contrast, soils that were oxic in the field showed microbial reduction of particulate TEAs and higher reactivity toward reduction of the TEAs. Both the microbial reduction of particulate TEAs and their reactivity toward reduction decreased over time; the decrease was accompanied by a decrease in carbon dioxide production. These results suggest that microbial respiration in the incubations was likely limited in part by the low redox reactivity of particulate TEAs. 